\title{
ON A TOPOLOGICAL GENERALIZATION OF A THEOREM OF TVERBERG
}

\author{
I. BÁRÁNY, S. B. SHLOSMAN AND A. SZÜCS
}

\section{Introduction}

Let $\Delta^{j}$ denote the $j$-dimensional simplex. The support of the point $x \in \Delta^{j}$ is the minimal face of $\Delta^{j}$ containing $x$. A face of $\Delta^{j}$ is understood to be closed.

The well-known theorem of Radon [3] can be formulated as follows.

Proposition 1. For any linear map $f: \Delta^{n+1} \rightarrow R^{n}$ there exist two disjoint faces $\Delta^{t}$ and $\Delta^{t_{2}}$ of $\Delta^{n+1}$ whose images $f\left(\Delta^{t_{1}}\right)$ and $f\left(\Delta^{t_{2}}\right)$ are not disjoint.

This proposition is generalised in [1].

Proposition 2. The statement of Proposition 1 holds for any continuous map $f: \Delta^{n+1} \rightarrow R^{n}$

Proposition 2 is a simple corollary of the following two statements.

Statement A. There exists a continuous map $g: S^{n} \rightarrow \Delta^{n+1}$ such that for every $x \in S^{n}$ the supports of $g(x)$ and $g(-x)$ are disjoint.

Statement B (Borsuk's and Ulam's antipodal theorem [2]). For any continuous map $h: S^{n} \rightarrow R^{n}$ there exists $x \in S^{n}$ with $h(x)=h(-x)$.

To see that the Statements A and B together imply Proposition 2 suppose that $f: \Delta^{n+1} \rightarrow R^{n}$ does not satisfy Proposition 2. Then the composition $f \circ g: S^{n} \rightarrow R^{n}$ would not satisfy Statement $B$ and this would be a contradiction.

Another generalization of Proposition 1 is proved in [5].

Proposition 3. For any linear map $f: \Delta^{N} \rightarrow R^{n}$, where $N=(p-1)(n+1)$, there exist $p$ pairwise disjoint faces $\Delta^{t_{1}}, \ldots, \Delta^{t_{p}} \subseteq \Delta^{N}$ such that $f\left(\Delta^{t_{1}}\right) \cap \ldots \cap f\left(\Delta^{t_{p}}\right)$ is nonempty.

The aim of this paper is to prove the following.

Theorem. Suppose $p$ is prime, $n \geqslant 1, N=(p-1)(n+1)$ and $f: \Delta^{N} \rightarrow R^{n}$ is a continuous map. Then there exist $p$ pairwise disjoint faces $\Delta^{t_{1}}, \ldots, \Delta^{t_{p}}$ of $\Delta^{N}$ such that $f\left(\Delta^{t_{1}}\right) \cap \ldots \cap f\left(\Delta^{t_{p}}\right)$ is nonempty.

We mention that if it were not for the restriction that $p$ be prime, then this theorem would be a common generalization of Propositions 2 and 3. We do not know whether the Theorem holds for any $p$ or not.

Received 17 May, 1979.

[J. London MATH. Soc. (2), 23 (1981), 158-164] 


\section{The scheme of the proof}

We shall deal with the odd primes only (for $p=2$ see [1]). The idea of the proof of the Theorem is the same as in Proposition 2 with only the change that in both Statements $\mathrm{A}$ and $\mathrm{B}$, instead of the sphere $S^{n}$, we shall take a $\mathrm{CW}$-complex $X=X_{n, p}$, and, instead of the antipodal map, we shall have the cyclic group $Z_{p}$ acting freely on $X$. The action of its generator is denoted by $\omega$.

Definition. Let us take $p$ disjoint copies of the $n(p-1)$-dimensional disc and identify their boundaries. This is the CW-complex $X_{n, p}$. The identified boundary, $S^{n(p-1)-1}$, is embedded into $X_{n, p}$ via

$$
i: S^{n(p-1)-1} \rightarrow X_{n, p}
$$

Suppose the cyclic group $Z_{p}$ acts freely on the sphere $S^{n(p-1)-1}$, and let $\omega$ denote the action of its generator. This map $\omega$ can be extended from $S^{n(p-1)-1}$ to $X_{n, p}$ as follows. If $(y, r, q)$ denotes the point of $X_{n, p}$ from the $q$-th disc with radius $r$ and $S^{n(p-1)-1}$ coordinate $y$, then put

$$
\omega(y, r, q)=(\omega y, r, q+1)
$$

where $q+1$ is reduced modulo $p$. Clearly, this map $\omega$ defines a free $Z_{p}$ action on $X_{n, p}$.

Note that on the odd dimensional sphere $S^{k}$ there always exists a free $Z_{p}$ action. So here we only need $p$ to be odd. In Section 4 we shall specify $\omega$.

We remark further that $X_{n, p}$ is defined for every $n, p \geqslant 1$. It is clear that $\operatorname{dim} X_{n, p}=n(p-1)$ and $X_{n, p}$ is $[n(p-1)-1]$-connected.

We shall prove the following two statements.

Statement $A^{\prime}$. There exists a continuous map $g: X \rightarrow \Delta^{N}$ such that for every $x \in X$ the supports of the points $g(x), g(\omega x), \ldots, g\left(\omega^{p-1} x\right)$ are pairwise disjoint.

Statement B'. For the map $\omega$ defined in Section 4 and for any continuous map $h: X \rightarrow R^{n}$ there exists an $x \in X$ such that $h(x)=h(\omega x)=\ldots=h\left(\omega^{p-1} x\right)$.

Clearly, the Theorem follows from Statements $\mathrm{A}^{\prime}$ and $\mathrm{B}^{\prime}$.

\section{The proof of Statement $A^{\prime}$}

We shall prove this statement for every odd $p$. We define the $\mathrm{CW}$-complex $Y_{N, p}$ as

$$
\begin{array}{r}
Y_{N, p}=\left\{\left(y_{1}, \ldots, y_{p}\right): y_{1}, \ldots, y_{p} \in \Delta^{N},\right. \text { and the supports } \\
\text { of } \left.y_{1}, \ldots, y_{p} \text { are pairwise disjoint }\right\} .
\end{array}
$$

Clearly, there exists a free $Z_{p}$ action on $Y_{N, p}$ : its generator maps $\left(y_{1}, \ldots, y_{p}\right) \in Y_{N, p}$ into $\left(y_{2}, \ldots, y_{p}, y_{1}\right) \in Y_{N, p}$.

Now the existence of the map $g: X \rightarrow \Delta^{N}$ of Statement $A^{\prime}$ is equivalent to the 
existence of a $Z_{p}$ equivariant map $G: X \rightarrow Y_{N, p}$. The existence of such a map follows from homotopy theory if $\operatorname{dim} X-1$ is not greater than the connectedness of $Y_{N, p}$. Indeed, given a $Z_{p}$ equivariant cell subdivision of the space $X$ one can construct an equivariant map $G: X \rightarrow Y_{N, p}$ by induction on the dimension of the cells in the following way.

Step 0. Choose a 0-cell from each orbit of 0-cells (that is vertices). Define the map $G$ on these vertices arbitrarily and extend this map to a $Z_{p}$ equivariant map of all vertices.

Step $k$. Suppose that $G$ has been defined on the $(k-1)$-skeleton of $X$. Choose a cell from each orbit of $k$-cells. The map $G$ is defined on the boundary of these cells. By the $(k-1)$-connectedness of $Y$ the map $G$ can be extended to the $k$-cells chosen from each orbit. Now define $G$ on the other $k$-cells to be $Z_{p}$ equivariant.

So in order to prove Statement $\mathrm{A}^{\prime}$ it suffices to prove the following.

Lemma 1. For all natural numbers $N$ and $p$ with $N \geqslant p+1$,

$$
\pi_{j}\left(Y_{N, p}\right)=0 \text { for } 1 \leqslant j \leqslant N-p .
$$

Proof. (For this elementary proof we are indebted to the referee. Our original proof used the Leray spectral sequence.) Let $0, \ldots, N$ denote the vertices of $\Delta^{N}$. The Cartesian power $\left(\Delta^{N}\right)^{p}$ has a natural structure as a cell complex, a typical cell being $\sigma_{1} \times \ldots \times \sigma_{p}$, with each $\sigma_{i}$ a face of $\Delta^{N}$. The cell is also described by the $p$-tuple $\left(A_{1}, \ldots, A_{p}\right)$, where $A_{i}$ is the set of vertices of $\sigma_{i}$. Those $p$-tuples where the $A_{i}$ are pairwise disjoint form a subcomplex (isomorphic to) $Y_{N, p}$.

In view of Hurewicz's theorem it suffices to prove that $\pi_{1}\left(Y_{N, p}\right)=0$ (this would imply that $\left.H_{1}\left(Y_{N, p}\right)=0\right)$ and that $H_{2}\left(Y_{N, p}\right)=\ldots=H_{N-p}\left(Y_{N, p}\right)=0$. The case where $p=1$ is trivial because $Y_{N, 1}$ is the same as $\Delta^{N}$. When $p$ is greater than one it is convenient to consider, for $i=0, \ldots, N$, that subcomplex $Y_{N, p, i}$ of $Y_{N, p}$ which one gets by requiring $A_{1}$ to be a subset of $\{i, i+1, \ldots, N\}$. Thus

$$
Y_{N, p}=Y_{N, p, 0} \supset Y_{N, p, 1} \supset \ldots \supset Y_{N, p, N} .
$$

We shall show that the groups in question vanish for every $Y_{N, p, i}$ and so in particular for $Y_{N, p}$.

The proof is by double induction, on $p$ and $N-i$. We assume that our assertion holds for $Y_{N^{\prime}, p^{\prime}, i^{\prime}}$ whenever either $2 \leqslant p^{\prime}<p$ or $p^{\prime}=p$ and $N^{\prime}-i^{\prime}<N-i$.

If $i=N$, then $Y_{N, p, i}=Y_{N, p, N}$ is homeomorphic to $Y_{N-1, p-1}$, and so we can assume that $i<N$.

In order to prove that $\pi_{1}\left(Y_{N, p, i}\right)=0$ it suffices to show that any given closed edge-path in the 1-skeleton of $Y_{N, p, i}$ can be deformed by homotopies in $Y_{N, p, i}$ until it lies in $Y_{N, p, i+1}$. Let $u_{1}, \ldots, u_{m}=u_{1}$ be the vertices of the given path. Thus each $u_{k}$ is described by a $p$-vector with distinct components from $0, \ldots, N$ (each $A_{i}$ is a singleton), and $u_{k+1}$ differs from $u_{k}$ in at most one component.

The deformation will be done in four steps, each step consisting of several small deformations on short subpaths.

In the first step one "separates" those neighbouring pairs $u_{k}, u_{k+1}$ for which $i+1$ 
occurs as second, third, ..., or $p$-th component in both $u_{k}$ and $u_{k+1}$. Clearly we can assume that $u_{k} \neq u_{k+1}$. Let $u_{k}=(\ldots, x, \ldots, i+1, \ldots)$ and $u_{k+1}=(\ldots, y, \ldots, i+1, \ldots)$, say with $x \neq y$, where only the changing component and the component equal to $i+1$ are indicated. Put $u_{k}^{\prime}=(\ldots, x, \ldots, z, \ldots)$ and $u_{k+1}^{\prime}=(\ldots, y, \ldots, z, \ldots)$ where $z$ is chosen among those elements of $\{0, \ldots, N\}$ which are not components of $u_{k}$ or $u_{k+1}$. In view of the assumption that $N \geqslant p+1$, such a number $z$ exists. It is easy to see that the deformation of the subpath $u_{k} u_{k+1}$ into $u_{k} u_{k}^{\prime} u_{k+1}^{\prime} u_{k+1}$ is a homotopy over the 2-cell $(\ldots,\{x, y\}, \ldots,\{z, i+1\}, \ldots)$ in $Y_{N, p, i}$. Let $v_{1}, \ldots, v_{1}$ be the path obtained by the first step.

In the second step one deletes each $v_{k}$ which has $i+1$ among its last $p-1$ components. In this case, as a result of the first step, $v_{k-1}=(\ldots, x, \ldots)$, $v_{k}=(\ldots, i+1, \ldots)$ and $v_{k+1}=(\ldots, y, \ldots)$, and $x \neq i+1, y \neq i+1$. It is clear that the deletion of $v_{k}$ is a homotopy over the 2-cell $(\ldots,\{x, y, i+1\}, \ldots)$ in $Y_{N, p, i}$ (or over a 1 -cell if $x=y$ ). Let $w_{1}, \ldots, w_{1}$ be the path obtained.

The third step is similar to the first one and consists of insertion of a pair of vertices of the form $(i+1, \ldots)$ between every pair $w_{k}=(i, \ldots), w_{k+1}=(i, \ldots)$. In the fourth step, which is similar to the second one, all vertices of the form $(i, \ldots)$ are deleted. This gives a path in $Y_{N, p, i+1}$ as desired.

It remains to prove that $H_{j}\left(Y_{N, p, i}\right)=0$ when $2 \leqslant j<N-p$. We compute the homology using the given cell complex. The boundary operator $\partial$ is defined on cells by

$$
\begin{aligned}
\partial(\sigma) & =\partial\left(A_{1}, \ldots, A_{p}\right) \\
& =\sum(-1)^{e(x)}\left(A_{1}, \ldots, A_{r(x)} \backslash\{x\}, \ldots, A_{p}\right) .
\end{aligned}
$$

Here the sum is taken over those $x$ in $A_{1} \cup \ldots \cup A_{p}$ which belong to an $A_{r}=A_{r(x)}$ with $\left|A_{r}\right| \geqslant 2$, and $e(x)$ is defined by

$$
e(x)=\left|A_{1}\right|+\ldots+\left|A_{r(x)-1}\right|+\mid\left\{y: y \in A_{r(x)} \text { and } y<x\right\} \mid .
$$

Now the cells are of four different types according to whether

(1) $i \in A_{1}$ and $\left|A_{1}\right|>2$,

(2) $i \in A_{1}$ and $\left|A_{1}\right|=2$,

(3) $\{i\}=A_{1}$,

(4) $A_{1} \subset\{i+1, \ldots, N\}$.

Now put $\sigma^{-}=\left(A_{1} \backslash\{i\}, A_{2}, \ldots, A_{p}\right)$ if $\sigma$ is of the first or the second type, $\sigma^{*}=\left(\{i\}, A_{2}, \ldots, A_{p}\right)$ if $\sigma$ is of the second type and $\sigma^{+}=\left(A_{1} \cup\{i\}, A_{2}, \ldots, A_{p}\right)$ if $\sigma$ is of the fourth type and $i \notin A_{2} \cup \ldots \cup A_{p}$. Let $C$ be a $j$-chain in $Y_{N, p, i}$. Now $C=C_{1}+C_{2}+C_{3}+C_{4}$ where $C_{h}$ is the sum of those cells of $C$ which are of the $h$-th type $(h=1,2,3,4)$, and clearly

$$
\partial C=\left(C_{1}+C_{2}\right)^{-}-\left(\partial\left(\left(C_{1}+C_{2}\right)^{-}\right)\right)^{+}-C_{2}^{*}+\partial C_{3}+\partial C_{4} .
$$

Assume now that $\partial C=0$. We must prove that $C$ bounds in $Y_{N, p, i}$ and we start by observing that $\left(\partial\left(\left(C_{1}+C_{2}\right)^{-}\right)\right)^{+}=0$, so that $\partial\left(\left(C_{1}+C_{2}\right)^{-}\right)=0$. Now $\left(C_{1}+C_{2}\right)^{-}$is a 
$(j-1)$-chain in $Y_{N, p, i+1}$, and it is even isomorphic to a $(j-1)$-chain in $Y_{N-1, p, i+1}$, as the vertex $i$ does not appear in it. Thus, by the hypothesis of induction, $\left(C_{1}+C_{2}\right)^{-}=\partial D$, where $D$ is a $j$-chain in $Y_{N, p, i+1}$, not involving the vertex $i$. (Here we have made use of the fact that $N>p+1$ for we are finished with the case where $N=p+1$.) This means that

$$
\partial\left(D^{+}\right)=D-\left(C_{1}+C_{2}\right)+\text { terms of the third type }
$$

and so $C+\partial\left(D^{+}\right)$has only terms of the third and fourth types. Put $C+\partial\left(D^{+}\right)=C_{3}^{\prime}+C_{4}^{\prime}$. Then $\partial C_{3}^{\prime}=\partial C_{4}^{\prime}=0$, as $\partial C=0$. But $C_{3}^{\prime}$ bounds in $Y_{N, p, i}^{*}$, because $H_{j}\left(Y_{N-1, p-1}\right)=0$, and $C_{4}^{\prime}$ bounds in $Y_{N, p, i}$ because $H_{j}\left(Y_{N, p, i+1}\right)=0$. This finishes the proof.

\section{Proof of Statement $B^{\prime}$}

First we shall specify the map $\omega: X_{n, p} \rightarrow X_{n, p}$. As we have seen, it is enough to specify $\omega: S^{n(p-1)-1} \rightarrow S^{n(p-1)-1}$. Now let $\theta: \prod_{1}^{p} R^{n} \rightarrow \prod_{1}^{p} R^{n}$ be defined by

$$
\theta\left(v_{1}, \ldots, v_{p}\right)=\left(v_{2}, \ldots, v_{p}, v_{1}\right)
$$

Put $D=\left\{(v, v, \ldots, v) \in \prod_{1}^{p} R^{n}: v \in R^{n}\right\}$. Then $\theta$ acts freely on $\prod_{1}^{p} R^{n} \backslash D$ (this is the point where we need $p$ to be prime). So $\theta$ acts freely on the unit sphere of the orthogonal complement of $D$ (relative to $\prod_{1}^{p} R^{n}$ ), or, what is the same thing, on the sphere $S^{n(p-1)-1}$. Now we define $\omega$ as the restriction of the map $\theta$ to this sphere. It is clear that $S^{n(p-1)-1}$ is $\theta$-invariant and it is a $\theta$-equivariant deformation retract of the space $\prod_{1}^{p} R^{n} \backslash D$.

Now we prove Statement $B^{\prime}$ with this $\omega$. Suppose, on the contrary, that there exists a map $h: X \rightarrow R^{n}$ for which Statement $\mathrm{B}^{\prime}$ does not hold. Then the image of the map $H: X \rightarrow \prod_{1}^{p} R^{n}$ defined by $H(x)=\left(h(x), h(\omega x), \ldots, h\left(\omega^{p-1} x\right)\right)$ is disjoint from the diagonal $D$. It is obvious that $H$ is equivariant, that is $H \omega=\theta H$.

Further, the injection $i: S^{n(p-1)-1} \rightarrow X$ is $\omega$-equivariant and, in view of the $[n(p-1)-1]$-connectedness of $X$, homotopic to zero. Thus the diagram

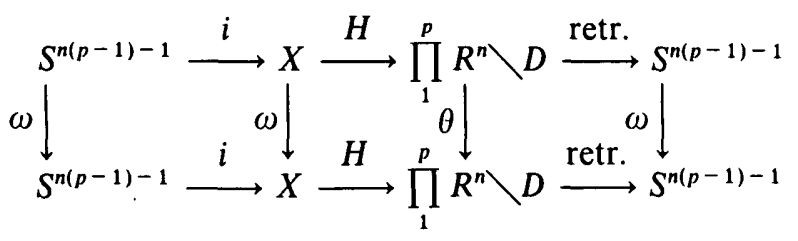


is commutative. Then the composition of the horizontal maps, $\xi: S^{n(p-1)-1} \rightarrow S^{n(p-1)-1}$, is equivariant and homotopic to zero. This implies that $\xi$ must have degree zero. But the following lemma will show that $\operatorname{deg} \xi \equiv 1 \bmod p$, thus providing a contradiction.

Lemma 2. Suppose that $k \geqslant 1, p \geqslant 2$ and we are given a free $Z_{p}$ action on the sphere $S^{k}$. Then an arbitrary equivariant map $\alpha: S^{k} \rightarrow S^{k}$ has degree 1 modulo $p$.

Remark. Note that here we do not need $p$ to be prime.

Lemma 2 is proved in [4; Theorem 8.3 , p. 42]. Here we present a simple proof.

Proof. Write $\theta$ for the action of the generator of $Z_{p}$ and choose a $\theta$-invariant cell subdivision on the sphere $S^{k}$. Since $\pi_{j}\left(S^{k}\right)=0$ for $j<k$ the restrictions of the map $\alpha$ and the identity map $S^{k} \rightarrow S^{k}$ to the $(k-1)$-skeleton of $S^{k}$ are equivariantly homotopic. Hence one can assume that $\theta$ coincides with the identity on the $(k-1)$-skeleton. (To see this more precisely one can use an argument from homotopy theory which is similar to the one in the proof of Statement $\mathbf{A}^{\prime}$.)

Let us consider the map $F: \bigcup_{j=1}^{p} S_{j}^{k} \rightarrow S^{k}$ of the disjoint union of $p$ copies of $k$-spheres $S_{1}^{k}, \ldots, S_{p}^{k}$ into the sphere $S^{k}$ defined by the formula

$$
F(x)= \begin{cases}\alpha(x) & \text { if } \quad x \in S_{1}^{k} \\ x & \text { otherwise }\end{cases}
$$

It is clear that $\operatorname{deg} F=\operatorname{deg} \alpha+p-1$.

The $\theta$-invariant cell subdivision of $S^{k}$ obviously has the property that the orbit of an arbitrary cell $\sigma$ consists of $p$ cells. (These are $\sigma, \theta(\sigma), \ldots, \theta^{p-1}(\sigma)$.)

Now let $\beta: S^{k} \rightarrow S^{k}$ be a continuous map which coincides with $\alpha$ on one of the $k$-cells of each orbit and with the identity on the others. Define the map

$$
G: \bigcup_{j=1}^{p} S_{j}^{k} \rightarrow S^{k}
$$

by

$$
G(x)=\beta \circ \theta^{j}(x) \quad \text { if } \quad x \in S_{j}^{k} \quad(j=1, \ldots, p) .
$$

Then on the one hand $\operatorname{deg} F=\operatorname{deg} G$ and on the other hand $\operatorname{deg} G \equiv 0 \bmod p$. This proves the lemma.

\section{References}

1. E. G. Bajmóczy and I. Bárány, "On a common generalization of Borsuk's and Radon's theorem”, Acta Math. Acad. Sci. Hungar., 34 (1979), 347-350.

2. K. Borsuk, "Drei Sätze über die $n$-dimensionale euklidische Sphäre", Fund. Math., 20 (1933), 177-190.

3. L. Danzer, B. Grünbaum and V. Klee, "Helly's theorem and its relatives", Convexity, Proceedings of Symposia in Pure Mathematics 7 (American Mathematical Society, Providence, 1963). 
4. M. A. Krasnoselsky and P. P. Zabrejko, Geometritsheskie zadatshi nelinejnogo analiza (Nauka, Moscow, 1975).

5. H. Tverberg, "A generalization of Radon's theorem", J. London Math. Soc., 41 (1966), 123-128.

Mathematical Institute of the Hungarian Academy of Sciences, 1053 Budapest,

Hungary.

Institute for Information Transmission Problems,

Moscow,

U.S.S.R.

József Attila University,

Bolyai Institute,

6720 Szeged,

Hungary. 